\title{
Anterior Cingulate Cortex Lesions Abolish Budget Effects on Effort-Based Decision-Making in Rat Consumers
}

\author{
Yue Hu (胡 悦), ${ }^{1}$ Marijn van Wingerden, ${ }^{2}{ }^{\circledR}$ Manuela Sellitto, ${ }^{1}$ Sandra Schäble, ${ }^{1}$ and Tobias Kalenscher ${ }^{1}$ \\ ${ }^{1}$ Comparative Psychology, Institute of Experimental Psychology, Heinrich Heine University Düsseldorf, Düsseldorf 40225, Germany, and \\ ${ }^{2}$ Department of Cognitive Science and Artificial Intelligence, Tilburg University, Tilburg 5037 AB, The Netherlands
}

Demand theory can be applied to analyze how animal consumers change their selection of commodities in response to changes in commodity prices, given budget constraints. Previous work has shown that demand elasticities in rats differed between uncompensated budget conditions in which the budget available to be spent on the commodities (e.g., the finite number of discrete operants to "purchase" rewards in two-alternative fixed-ratio schedules) was kept constant, and compensated budget conditions in which the budget was adjusted so that consumers could potentially continue to obtain the original reward bundles. Here, we hypothesized that rat anterior cingulate cortex (ACC) was necessary to produce this budget effect on demand elasticities. We applied excitotoxic or sham lesions to ACC in rats performing an effort task in which the prices of liquid vanilla or chocolate rewards (the effort required to obtain rewards) and the budget (the total number of operants) was manipulated. When reward prices changed, and the budget was compensated, all rats adjusted their demand for chocolate and vanilla accordingly. In sham-lesioned rats, changes in demand were even more pronounced when the budget was not compensated for the price changes. By contrast, ACC-lesioned animals did not show this additional budget effect. An indepth comparison of the rats' choice patterns showed that, unlike sham rats, ACC-lesioned animals failed to maximize session-bundle utility after price/budget changes, revealing deficits in higher-order choice-strategy adaptations. Our results suggest a novel role of ACC in considering purchasing power during complex cost-benefit value computations.

Key words: anterior cingulate cortex; budget; cost-benefit decisions; demand elasticity; neuroeconomics; rodents

Significance Statement

Anterior cingulate cortex (ACC) is important for allocating effort in cost-benefit calculations in animals and humans. Economic theory prescribes that the value of the costs in cost-benefit analyses not only depends on the net nominal costs required to purchase a reward, but also on the available budget resources, i.e., on the budget's "purchasing value." We asked whether ACC, a region implicated in effort-based decision-making and reward comparisons, is required for computing the value of effort relative to a budget constraint. Applying demand theory to describe rat choices in a rodent effort allocation task with varying effort prices and budgets, we show that ACC integrity was necessary for computing purchasing power, a core variable in economic choice theory.

\section{Introduction}

An old Chinese proverb says “物以稀为贵”: when a thing is scarce, it becomes precious. This proverb captures the essence of the economic principles of supply, demand and value, stating

\footnotetext{
Received Sep. 29, 2020; revised Feb. 26, 2021; accepted Feb. 27, 2021

Author contributions: Y.H., M.v.W., and T.K. designed research; Y.H. performed research; M.v.W., M.S., S.S., and T.K. contributed unpublished reagents/analytic tools; Y.H., M.v.W., M.S., and T.K. analyzed data; Y.H., M.v.W., M.S., S.S., and T.K. wrote the paper.

This work was supported by Deutsche Forschungsgemeinschaft Grants DFG-HU 2690/2-1 (to Y.H.) and DFG KA 2675/5-3 (to T.K.). We thank John H. Kagel for his comments on an earlier version of the manuscript. We thank Brian M. Sweis for his suggestion at SfN on the analyses.

*Y.H. and M.v.W. contributed equally to this work.

The authors declare no competing financial interests.

Correspondence should be addressed to Yue Hu at yue.hu@hhu.de.

https://doi.org/10.1523/JNEUROSCI.2541-20.2021

Copyright $\odot 2021$ the authors
}

that the demand of a good depends on its subjective value, its price, and the available budget. Yet, prices are subject to inflation. If the individual income (i.e., the budget) remains fixed, the total amount of priced commodity bundles that consumers can afford, i.e., the income's purchasing power, decreases. However, adjusting individual income for inflation restores purchasing power and allows the consumers to reselect the same bundle of goods as before. Hence, when compensating the budget for inflation, demand becomes less elastic. This phenomenon is termed the budget effect on demand elasticity, which has been widely observed in human consumer behaviors (Deaton and Muellbauer, 1980) but, interestingly, also in animal consumers, such as rats (Kagel et al., 1975, 1981) and capuchin monkeys (Chen et al., 2006).

A previous study from our group replicated the budget effects on demand elasticity in a task adopted for rats (van Wingerden 
et al., 2015). Rats expended variable numbers of nose pokes (NPs; "prices") to obtain differently priced chocolate or vanilla milk rewards which they were able to "buy" from a fixed daily budget of NPs. We found that the demand for milk was strongly sensitive to changes in milk prices, but, importantly, demand was considerably less elastic when the daily NP budget was adjusted to compensate for the price changes. An in-depth analysis of dynamic choice selection revealed that rats engaged in a genuinely budget-dependent valuation of the priced commodities. In other words, the value of one unit of milk at a given price depended on the available budget. Thus, rats evaluated NP effort costs not only by their nominal value (i.e., the net number of NPs required to obtain a reward) but also with respect to their purchasing power (i.e., how much milk can be purchased given its price and the available NP budget).

Despite extensive behavioral evidence for the budget effect on demand elasticity, our knowledge of the underlying neural mechanisms is still scarce. One possible candidate region is the anterior cingulate cortex (ACC). ACC is known to be important for value integration to guide effort-based decisions in humans (Croxson et al., 2009; Prévost et al., 2010; Klein-Flügge et al., 2016), monkeys (Kennerley and Wallis, 2009), and rodents (Walton et al., 2002; Rudebeck et al., 2006; Porter et al., 2019). For example, ACC has been implicated in predicting effort cost requirements (Vassena et al., 2014), processing energetic (Laughlin et al., 1998) and opportunity costs (Kurzban et al., 2013), and integrating (Hillman and Bilkey, 2010, 2012) and tracking (Kolling et al., 2012) costs and benefits. However, it is less clear whether ACC engages in the evaluation of the nominal cost of effort, or whether computes its real value by considering an effort budget constraint during cost-benefit calculations. We, therefore, asked whether ACC is important for mediating the budget effects on demand elasticity in rat consumers.

To address this question, we applied bilateral neurotoxic lesions to the dorsal part of rat ACC (dACC) and compared their economic choices to those of sham-lesioned control rats in our rodent consumer demand task. All rats adjusted their demand for milk to changes in milk prices. However, unlike rats in the sham group, ACC-lesioned animals adjusted their demand to changes in milk prices independent on whether their budget was compensated for the price changes or not, suggesting that they failed to integrate the budget constraint into their cost-benefit value computation. In-depth analyses showed that sham, but not lesioned, rats changed their choice-strategy in a budget-dependent fashion, thus offering insights into potential mechanisms how rats consider effortbudgets. Our findings expand our understanding of role of ACC in high-level cost-benefit decision-making.

\section{Materials and Methods}

\section{Subjects}

Twenty-four adult male Long-Evans rats (Charles River Laboratories) were used for this experiment. All animals weighed between 280 and $350 \mathrm{~g}$ at the beginning of the experiments and had not been used in any previous testing. Rats were housed in a group of three and under an inverted $12 / 12 \mathrm{~h} \mathrm{light/dark}$ cycle, at a temperature of $22 \pm 2^{\circ} \mathrm{C}$ and humidity-controlled (60\%) colony room. Throughout the experiment, all animals received food deprivation with water available ad libitum in the home cages and were kept at $85 \%$ of free-feeding body weight. All animal procedures were conducted in accordance with the German Welfare Act and were approved by the local authority LANUV (Landesamt für Natur-, Umwelt- und Verbaucherschutz North RhineWestphalia, Germany). One animal was excluded from the final analysis because it did not learn the task, another animal passed away after surgery, and one further rat in the lesion group was excluded because of incomplete lesion of dACC. The final analysis included twelve animals in the lesion group and nine animals in the sham group.

\section{Apparatus}

The experiment was conducted in an operant chamber $(28 \times 23 \times$ $23 \mathrm{~cm}$; Med Associates Inc.) under red light. The front panel of each chamber was equipped with three NP units arranged horizontally. All NP holes contained photograph beams that were able to detect and signal when rats entered the hole with their snouts. On the opposite side of the chamber, the back panel was equipped with one central house light, two reward bottle access holes with infrared photograph beams to detect head entry as well as two reward lights located above each access hole. Once the animal met the fixed ratio requirement (i.e., a given number of NPs), one (forced-choice trials; see below) or two (free-choice trials) reward lights were turned on, and motorized drives lowered the reward dispenser bottle through the hole to make liquid reward accessible for 2 s. After the start of the experiment, the house light was turned off until the end of the session. All inputs, outputs, and events were timestamped, controlled, and recorded by the software MedPC (Med Associates Inc.), and stored for offline analysis.

\section{Behavioral task}

Budget and price conditions

The task was an adapted version of van Wingerden et al. (2015) and is illustrated in Figure 1. Rats were trained to choose between vanilla and chocolate milk rewards (chocolate and vanilla soymilk, diluted with water; chocolate milk, 2:3 water; vanilla milk, 1:3 water). They traded instrumental effort (in the following referred to as the price of a reward: the number of NPs necessary to obtain a reward) for rewards while their budget (the total number of NPs available per session that could be distributed between two rewards) was constrained. Animals went through five conditions following an A-B-A-B'-A block design (see below in Session and block structure), so that each price-budget condition was preceded and followed by a baseline phase. In the baseline phase, each reward had a price of 2 NPs (rats had to keep their nose in the hole for $100 \mathrm{~ms}$ to count as one NP), and animal consumers had a fixed budget of $n=80$ NPs they could spend on vanilla or chocolate reward. We measured the baseline demand for chocolate and vanilla milk, given this price and this limited budget. Next, we changed the price of vanilla and chocolate milk to 1 and 4 NPs, respectively, and estimated the change in demand for both commodities (i.e., demand elasticity). Importantly, we also manipulated the budget. In the uncompensated budget condition, the budget remained at 80 sNPs after implementing the price changes; that is, rats had to complete $80 \mathrm{NPs}$, as in the baseline sessions. In the compensated budget condition, the budget was compensated following Slutsky's demand equation (Slutsky and Ragusa, 2012) to make sure animal consumers could continue to purchase the same commodity bundle as in the preceding baseline condition. The budget compensation was done independently for each rat, based on their individual baseline choices. For example, assume that a rat spends its $80 \mathrm{NPs}$ at baseline to purchase 30 units of chocolate and 10 units of vanilla. To keep purchasing this commodity bundle after changing the chocolate price to 4 NPs, and the vanilla price to $1 \mathrm{NP}$, the budget would have to be increased to $130 \mathrm{NPs}$ in the compensated budget condition $(30 \times 4+10 \times 1=130)$ to compensate the price changes. That is, with a new budget of $130 \mathrm{NPs}$, the rat could purchase the same reward bundle obtained during baseline, despite the change in reward prices.

\section{Trial structure}

Each trial began with the activation of the central NP hole on the front panel, indicated by illumination. Rats initiated the trial by making a single NP for $200 \mathrm{~ms}$, followed by the activation and illumination of one (in forced-choice trials) or both (in free-choice trials) lateral NP holes. Rats were required to meet a condition-specific and choice-specific fixed ratio requirement (a variable number of $100-\mathrm{ms}$ NP responses; see above, Budget and price conditions) to obtain a chocolate or vanilla reward. NP responses in one of the two lateral holes yielded chocolate milk reward, 


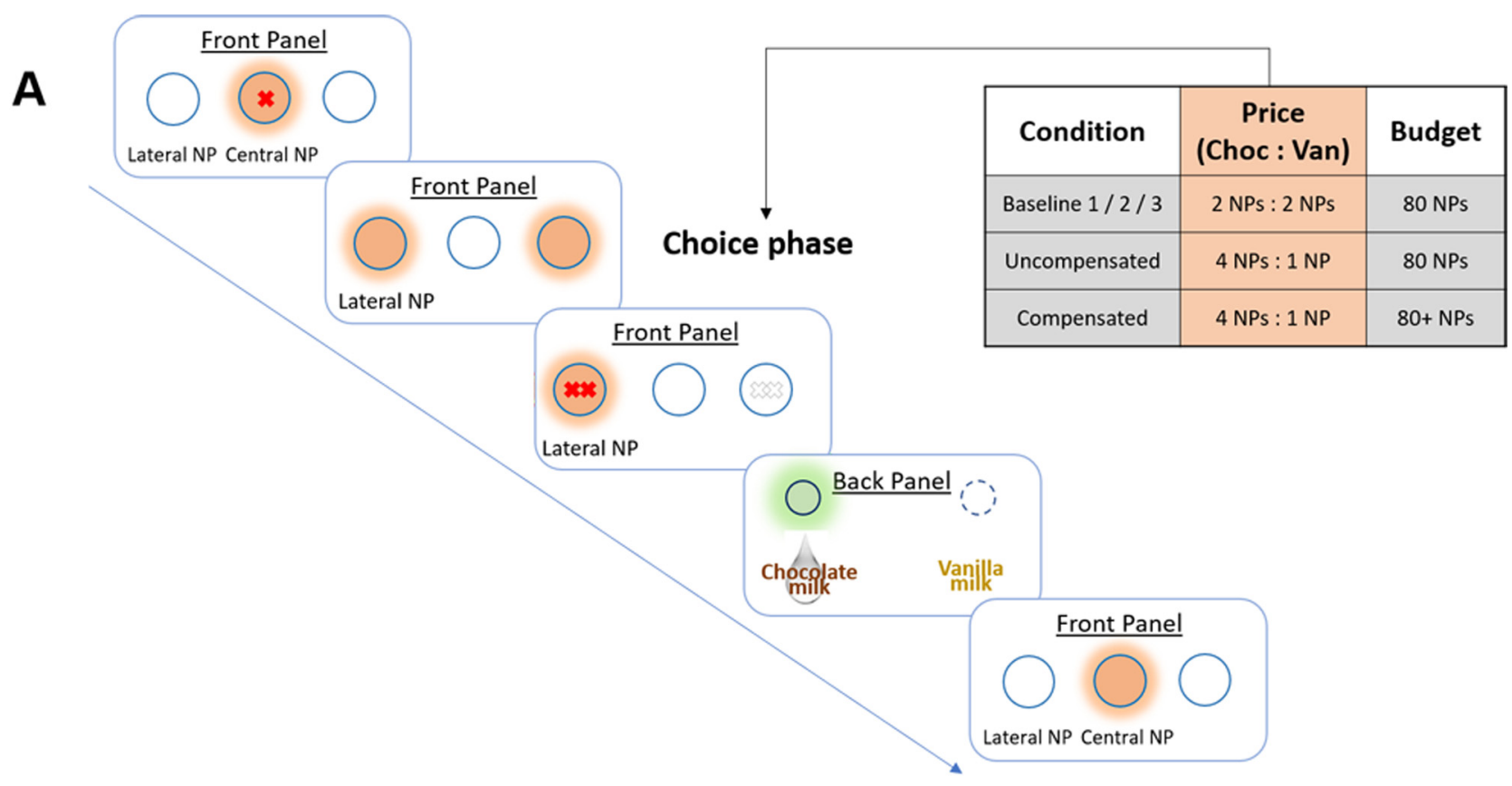

B

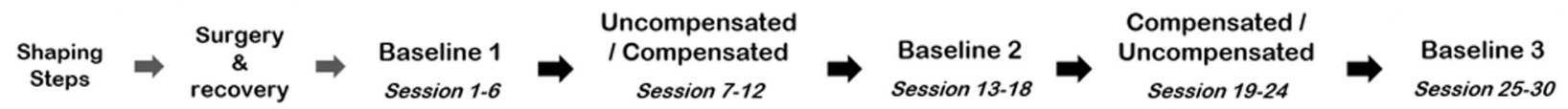

Figure 1. Outline of rat effort-budget task and timeline of experimental manipulations. $\boldsymbol{A}$, Schematic illustration of the operant chambers and the basic structure of a single trial. Rats initiated a trial by making a single NP to the activated central hole (activation indicated by a NP hole light; here, orange color), followed by the activation of both lateral NP holes. Rats were required to meet a condition-specific and choice-specific fixed ratio requirement, here indicated by the number of crosses, in one of the two lateral NP holes to obtain chocolate or vanilla rewards, delivered at the back panel. The table inset illustrates the ratio requirement: at baseline, the price for chocolate (Choc) and vanilla (Van) was fixed at 2 NPs and the budget the rats could spend to purchase chocolate or vanilla was fixed at $80 \mathrm{NPs}$. In the uncompensated budget condition, the price of chocolate was increased to 4 NPs, and the price of vanilla was decreased to $1 \mathrm{NP}$, the budget was kept at $80 \mathrm{NPs}$. In the compensated budget condition, the price change of the rewards was identical to the uncompensated budget condition, but the budget was adjusted following the Slutsky equation (see main text) to compensate the price changes and allow consumption of the baseline bundle of chocolate and vanilla. $\boldsymbol{B}$, Session structure. After pretraining, shaping, surgery and one-week recovery, rats performed the effort-budget task. Rats were trained on six consecutive days in each condition, with $1 \mathrm{~d}$ of rest between conditions. The order of two price/budget conditions was pseudorandomized across animals.

delivered in the back panel, responses in the other lateral hole yielded vanilla milk rewards. Rewards were presented for $2 \mathrm{~s}$, followed by an intertrial interval (ITI) of variable duration such that each trial lasted exactly $32 \mathrm{~s}$. Central and lateral NP holes were activated for a maximum period of $10 \mathrm{~s}$, or until rats responded. Failure to respond within $10 \mathrm{~s}$ was followed by ITI and a start of a new trial, i.e., re-activation of the central NP hole.

The NP side to reward type assignment was counterbalanced across sessions for each rat. Each session (see below, Session and block structure) always started with eight forced-choice trials, four on each side in a pseudorandom order, to allow animals to sample the reward and associated ratio requirements, followed by a variable number of free-choice trials. The forced-choice trials were not counted into the available budget in each session; i.e., rats could spend their full session-budget after having completed the forced-choice trials. A session was terminated until the animals spent the entire budget, or after $60 \mathrm{~min}$. In the baseline sessions, rats had a budget of $80 \mathrm{NP}$; each baseline session, hence, consisted of 40 trials given the equal price of 2 NPs per reward. In the price/budget conditions, the number of trials in each session varied across rats and depended on the respective budget and individual choices. For example, if a rat consumed 10 chocolate units (worth $40 \mathrm{NPs}$ ) and 40 vanilla units (worth $40 \mathrm{NPs}$ ) in the uncompensated budget condition, it would have spent $80 \mathrm{NPs}$ and would have completed 50 trials. For data analysis, we included only those sessions where at least $85 \%$ of the budget was spent. The percentages of sessions that were excluded from analysis were $4 \%$ for the lesioned and $4.6 \%$ for the sham animals.
Session and block structure

The experiment consisted of five blocks à six sessions (Fig. 1, table inset, $B$ ); as indicated above, the first block was always the baseline condition, followed by a block with higher chocolate and lower vanilla prices and either compensated or uncompensated budget, followed by the second baseline block, followed by another block with altered reward prices and compensated/uncompensated budget, followed by the third baseline block. The order of compensated versus uncompensated budget conditions was pseudorandomized and counterbalanced across rats.

Rats were trained on six consecutive days per block, one session per day, with $1 \mathrm{~d}$ of rest between blocks. Before testing in the final task, and before surgery, rats were pretrained in a five-step shaping procedure identical to (van Wingerden et al., 2015). Rats were allowed to recover for one week after surgery before proceeding to the final task.

\section{Data analysis}

To calculate proportions of chocolateor vanilla choices, and reward preferences, the number of choices for each reward was counted within each session, and averaged across bins of two sessions, separate for each condition and rat. The binning of sessions in blocks of two sessions was convenient to reduce decision noise across sessions.

We expressed a change in demand for chocolate and vanilla in the different baseline, price, and budget conditions with demand elasticity estimates (Kagel et al., 1981). Demand elasticity $(\varepsilon)$ is an index of price sensitivity and can be calculated using linear regression on the log-transformed number-of-choices/price-ratio pairs as given by the equation: 


$$
\log q_{i}=\log \alpha+\varepsilon \log \left(\frac{p_{i}}{p_{j}}\right)
$$

where $q_{i}$ indicates the quantity of reward (1) chosen, $p_{i} / p_{j}$ the price ratio for reward (1) over reward (j) per price/budget regime and $\alpha$ is a constant. Thus, this demand elasticity estimate quantifies how consumers change their consumption of a given reward as a function of its price and the available budget. More negative $\varepsilon$ parameters indicate a stronger change in demand in response to price and/or budget changes from baseline prices/budgets. We estimated $\varepsilon$ individually for each rat, session and condition, using linear regression. To facilitate the comparisons between budget conditions, we set each rat's baseline chocolate consumption to the median value of all their baseline sessions, and not local baselines, so that changes in demand elasticity are only dependent on the commodity choices in the compensated or uncompensated budget conditions, and not confounded by small differences in baseline choices. Repeating the same analyses with demand elasticity values computed from local, instead of median, baselines yielded similar results (see below).

We additionally computed cross-price elasticity. Cross-price elasticity estimates the level by which one reward is substituted, or complemented, by another reward, given the rewards' price changes. Thus, in a two-reward choice setting, cross-price elasticity of one reward indicates how strongly the consumption of this reward changes with the change of price of the other reward. Here, positive cross-price elasticity for vanilla means that vanilla substitutes for chocolate (the consumption of vanilla increases in response to the increase in chocolate prices), cross-price elasticity of zero for vanilla means that rewards are independent (the change in chocolate price does not affect vanilla consumption), and negative cross-price elasticity for vanilla implies that the rewards complement each other (the consumption of vanilla decreases in response to the increase in chocolate prices). Cross-price elasticity was calculated similarly to demand elasticity:

$$
\log q_{i}=\log \alpha+\varepsilon_{c} \log \left(\frac{p_{j}}{p_{i}}\right)
$$

thus, we express the log-transformed quantity consumed of commodity $i$ as a function of the log-transformed price-ratio/number-ofchoices pairs. The main difference to Equation 2 is that cross-price elasticity $\varepsilon_{c}$ uses the reciprocal of the price-ratio of Equation 1. In the present task, the cross-price elasticity of one commodity is identical to the sign-reversed price-elasticity of this commodity.

Choice data and demand elasticity were analyzed using a mixed ANOVA with Bonferroni correction for multiple comparisons. For the analysis of the choice proportions, we conducted an ANOVA with the between-subject factor lesion (sham vs lesion), and the within-subject factor budget condition (baselines, uncompensated budget, and compensated budget). For the analysis of demand elasticity, we considered the between-subject factor lesion (sham vs lesion), and the within-subject factor budget condition (uncompensated budget vs compensated budget). For the analysis of cross-price elasticity, we considered the within-subject factors budget condition (uncompensated vs compensated budget) and the between-subject factor lesion (lesion vs sham). All frequentist analyses are complemented by Bayesian ANOVAs, both were performed in JASP (Morey et al., 2016; JASP Team J, 2019). Thus, in addition to standard frequentist p-values, we also report the Bayes Ffactors (BFs) for each ANOVA and post hoc $t$ test. For two-factorial analysis, we report $\mathrm{BF}_{10}$ to quantify the relative evidence in favor of the best model compared with the null model and the inclusion BF $\left(\mathrm{BF}_{\text {incl }}\right)$ across matched models to quantify the contribution of this particular factor in improving the predictive performance of the model (Keysers et al., 2020). For two-sided post hoc $t$ tests, we use $\mathrm{BF}_{10}$ to quantify the likelihood of evidence in favor of the hypothesis H1 (i.e., two samples are not equal); for one-sided post hoc $t$ test, we report $\mathrm{BF}_{-0}$ to quantify the likelihood of evidence in favor of the hypothesis $\mathrm{H} 1$. Conventionally, we consider $\mathrm{BF}_{\mathrm{s}}$ values $>3$ as substantial evidence in favor of the alternative model, $1<\mathrm{BF}_{\mathrm{s}}<3$ as anecdotal evidence to support the model, and $\mathrm{BF}_{\mathrm{s}}<1$ suggests a lack of effect (Lee and Wagenmakers, 2014).

The linear modeling of categorical variables (group, condition and block) and the continuous predictor "baseline chocolate preference" (see below) on cross-price elasticity for vanilla was implemented in a mixedeffect model, with random effects for condition and block, per animal, using the NLME R-package (Pinheiro et al., 2007). Robust slope statistics were extracted using the "sfsmisc" package (Maechler et al., 2020) on reduced robust linear models (rlm from the MASS package; Ripley et al., 2013) without a repeated-measures component (i.e., when restricting data to a single block/condition combination).

\section{Neurotoxic lesions}

Neurotoxic lesions of bilateral ACC were made by intracerebral infusions of quinolinic acid $(0.09 \mathrm{M})$ in phosphate buffer vehicle using the following parameters (Rudebeck et al., 2006): site 1, AP $+2.3 \mathrm{~mm}$, $\mathrm{ML} \pm 0.5 \mathrm{~mm}, \mathrm{DV}-1.5 \mathrm{~mm}(0.2 \mu \mathrm{l})$; site $2: \mathrm{AP}+1.6 \mathrm{~mm}, \mathrm{ML} \pm 0.5 \mathrm{~mm}$, $\mathrm{DV}-2.0 \mathrm{~mm}(0.3 \mu \mathrm{l})$; site $3: \mathrm{AP}+0.9 \mathrm{~mm}, \mathrm{ML} \pm 0.5 \mathrm{~mm}, \mathrm{DV}-0.2 \mathrm{~mm}$ $(0.2 \mu \mathrm{l})$; site $4: \mathrm{AP}+0.2 \mathrm{~mm}, \mathrm{ML} \pm 0.5 \mathrm{~mm}, \mathrm{DV}-2.0 \mathrm{~mm}(0.2 \mu \mathrm{l})$. Infusions were made at a rate of $0.1 \mu \mathrm{l}$ every $30 \mathrm{~s}$ with a 30 -s interval between injections, needles remained in place for 2-min postinfusion to ensure the substance diffused away from the injection site. The sham lesion was conducted in the same way, except that an equal amount of phosphate buffer solution was injected instead of quinolinic acid.

The accuracy of the lesions was histologically assessed after task completion. Rats were transcardially perfused with $4 \%$ paraformaldehyde in $0.1 \mathrm{~m}$ phosphate buffer and brains were stored in the fixation solution until further processing. Coronal sections were cut at a thickness of 45 $\mu \mathrm{m}$ using a vibratome (Leica) and stained with $1 \%$ cresyl violet perchlorate to visualize the location of the lesion. Lesions were identified as an accumulation of apoptotic cells or a clear trace of tissue damage, verified by a blind experimenter. The locations of the lesions are superimposed onto stereotaxic maps of the rat brain (Paxinos and Watson, 2006). An example and a schematic reconstruction of the lesion are shown in Figure 2.

\section{Results}

\section{Hypotheses}

We hypothesized that (1) there is a main effect of milk price (effort) on commodity choice, i.e., all rats show effort discounting and adjust their demand of chocolate and vanilla to the price changes of these commodities, (2) there is an effect of budget on demand elasticity (van Wingerden et al., 2015), i.e., demand elasticity of chocolate and vanilla milk in response to price changes are different in the compensated budget than the uncompensated budget condition; (3) dACC lesions selectively abolish the budget effects on demand elasticity; i.e., unlike in control animals, demand elasticity in dACC-lesioned rats should be similar between the compensated and the uncompensated budget condition; (4) dACC lesion effects on demand are the consequence of a higher-order learning deficit: dACC-lesioned animals would fail to optimize their individual choice strategy to maximize the utility derived from the bundle of rewards obtained in a session.

\section{dACC lesions abolish the budget effect on demand elasticity}

To test these hypotheses, we first focused on the choice data from the last two sessions (sessions 5 and 6) within each budget condition where the rats' choice patterns reached a stable state (van Wingerden et al., 2015), i.e., we ignored learning effects in the first four sessions after price/budget changes for now (see below for choice data across all sessions). For ease of exposition, we focus on chocolate choices only (see below for a more detailed analysis on chocolate and vanilla choices). A mixed ANOVA revealed a significant main effect of budget condition (baselines vs compensated vs uncompensated; $F_{(2,19)}=49.662, p<0.01$, 
A

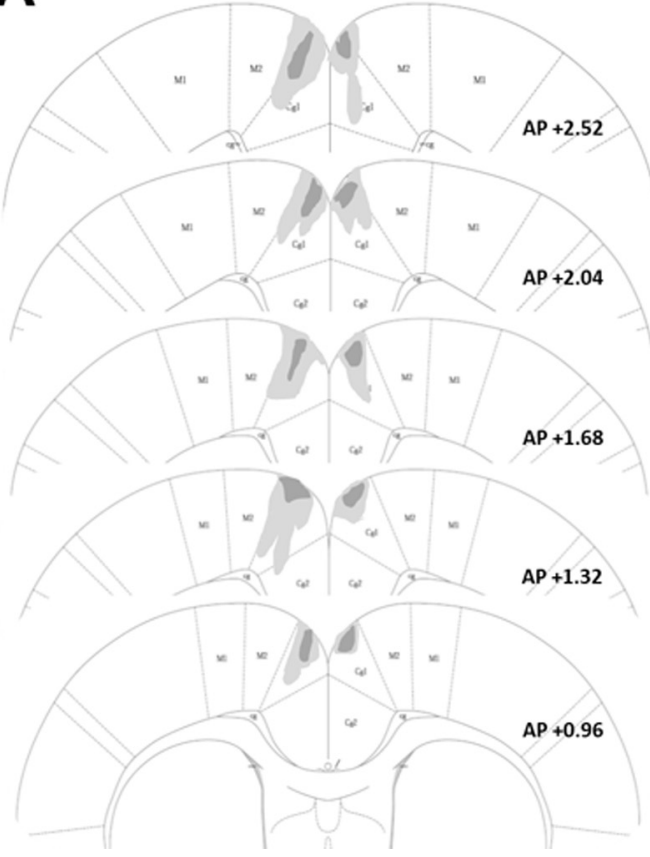

B
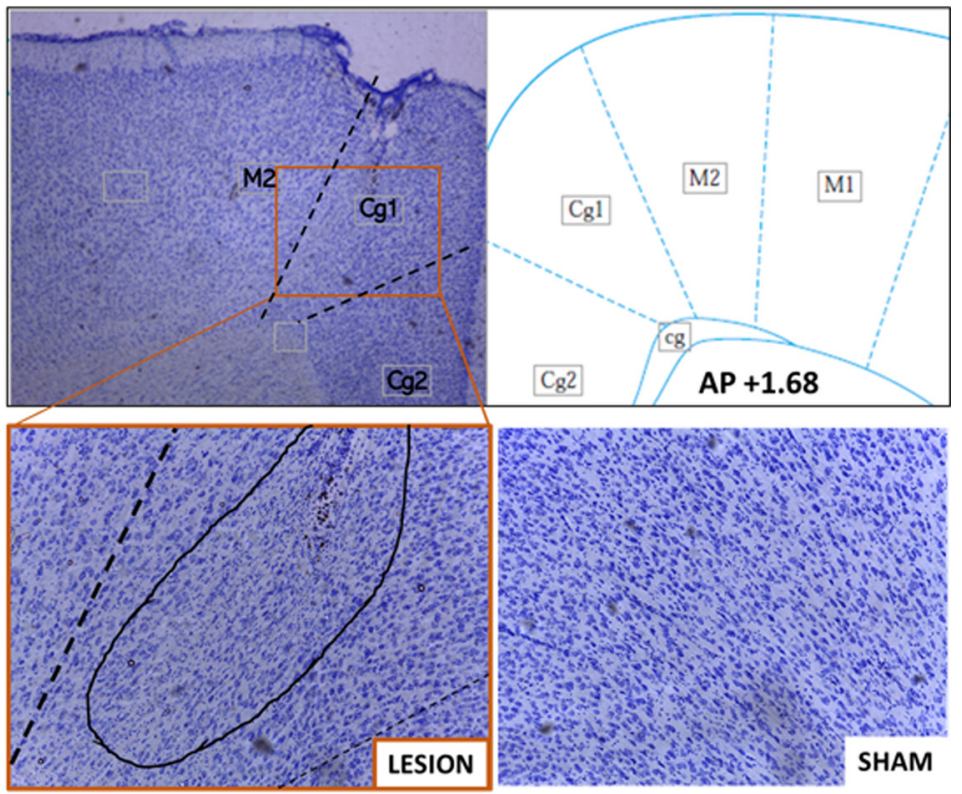

Figure 2. Schematic and photomicrograph representation of ACC lesions. A, Schematic representation of ACC lesion placements from anterior to posterior. We found the most overlapping lesions in the dorsal regions (dACC, $\mathrm{Cg} 1)$ spreading to the motor cortex (M2) in the anterior regions. $\boldsymbol{B}$, An example brain slice of dACC lesion and sham group. Light color, maximum lesioned area; dark color, minimum area. Pictures are adapted from the atlas of Paxinos and Watson (2006).

partial $\eta^{2}=0.723$ ) on the proportion of chocolate choices as well as a significant budget $\times$ lesion (sham vs lesion) interaction effect on chocolate choices $\left(F_{(2,19)}=6.029, p=0.005\right.$, partial $\eta^{2}=$ 0.241). We further confirmed the robustness of these result in a Bayesian repeated-measures ( $\mathrm{rm}$ )ANOVA where we found that a full model containing budget condition, lesion and budget condition $\times$ lesion is the best model $\left(\mathrm{BF}_{10}=1.411 \mathrm{e} 10\right)$. Breaking down these main and interaction effects (Fig. $3 A$ ), in the baseline condition, when rewards were equally priced ( rice $_{\mathrm{choc}}=2$, price $\left._{\text {van }}=2\right)$, sham and lesioned rats exhibited similar preferences for chocolate (\%Choice_choc Sham_baseline $_{\text {SC }}=0.659 \pm 0.024, \%$ Choice_choc Lesion_baseline $=0.604 \pm 0.019, t_{(19)}=-0.920$, $\left.p=0.369, \mathrm{BF}_{10}=0.53\right)$, indicating that baseline preferences for chocolate were not affected by dACC lesions. Consistent with our first hypothesis, in the two budget conditions when the price of chocolate milk increased, and vanilla milk became cheaper

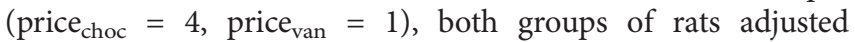
their consumption pattern and turned to purchasing less chocolate, compared with their baseline chocolate preferences $\left(\%\right.$ Choice_choc ${ }_{\text {Sham_Uncompensated }}=0.206 \pm 0.043, t_{(8)}=-8.843$,

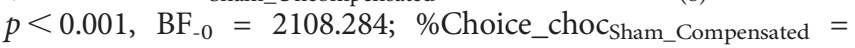
$0.343 \pm 0.052, t_{(8)}=-4.942, p<0.001, \mathrm{BF}_{-0}=46.014 ; \%$ Choice_choc Lesion_Uncompensated $=0.362 \pm 0.037, t_{(11)}=-4.956$, $p=0.001, \mathrm{BF}_{-0}=163.807 ; \%$ Choice_choc Lesion_Compensated $_{\text {L }}=$ $\left.0.272 \pm 0.045, t_{(11)}=-8.508, p<0.001, \mathrm{BF}_{-0}=10588.255\right)$. This suggests that all rats responded to the price changes and showed effort discounting by selecting chocolate less when the effort required to obtain it increased. Furthermore, in line with our second hypothesis, sham lesioned rats further reduced their chocolate consumption in the uncompensated compared with the compensated budget condition $\left(t_{(8)}=-2.497, p=0.019, \mathrm{BF}_{-0}=\right.$ $4.45)$, replicating our previous results (van Wingerden et al., 2015) that budget matters for the selection of priced rewards. This is notable since prices and rewards were identical in the compensated and uncompensated budget conditions, yet sham rats selected different reward bundles between budget conditions. Hence, the budget effect on choice cannot be explained by effort (price) discounting per se because chocolate prices were identical between conditions; this effect is more compatible with the notion that sham rats somehow processed the purchasing value of their NP budget. Importantly, in support of our third hypothesis, the budget effect on chocolate choices was not observed in dACC-lesioned animals: the difference in the proportion of chocolate choices between the uncompensated and the compensated budget conditions did not reach significance in the lesioned animals $\left(t_{(11)}=1.722, p=0.113, \mathrm{BF}_{-0}=0.126\right)$. Further post hoc tests revealed that, in the uncompensated budget condition, lesioned rats selected the expensive chocolate milk more often than sham rats $\left(t_{(19)}=2.770, p=0.012, \mathrm{BF}_{10}=\right.$ 4.477), but there was no significant difference between lesioned and sham rats in their chocolate selection in the compensated budget condition $\left(t_{(19)}=-1.036, p=0.313, \mathrm{BF}_{10}=0.574\right)$. Put together, these results support our third hypothesis, indicating that dACC lesions selectively diminished the budget effects on chocolate selections.

To further illustrate the diminished budget effects on choice after dACC lesions, we calculated demand elasticity (Fig. 3B). A rmANOVA on demand elasticity for chocolate revealed a significant main effect of budget condition on demand elasticity $\left(F_{(1,19)}=16.952, p<0.001\right.$, partial $\left.\eta^{2}=0.484\right)$ as well as a significant interaction effect between budget condition and lesion group $\left(F_{(1,19)}=8.838, p=0.008\right.$, partial $\left.\eta^{2}=0.312\right)$. A Bayesian rmANOVA further confirmed that a full model containing budget condition, lesion and budget condition $\times$ lesion outperforms models with less factors $\left(\mathrm{BF}_{10}=416.032\right)$. Demand elasticity of chocolate in the sham group differed significantly between the two budget conditions ( $\varepsilon_{\text {Choco_Sham_Compensated }}=-0.371 \pm$ $0.105, \varepsilon_{\text {Choco_Sham Uncompensated }}=-0.889 \pm 0.119, t_{(8)}=-4.69$, $\left.p<0.001, \mathrm{BF}_{10}=41.347\right)$. Importantly, this difference in demand elasticity for chocolate between the compensated and the 
A

\%Choice of Chocolate Milk

追 Baseline 追 Uncompensated 追 Compensated

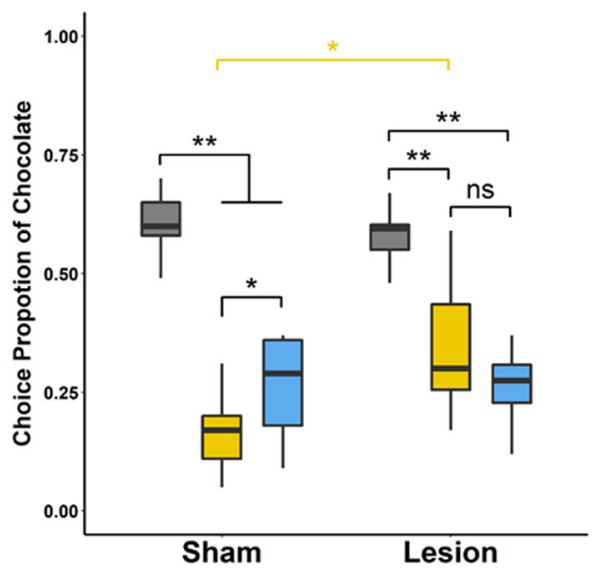

C

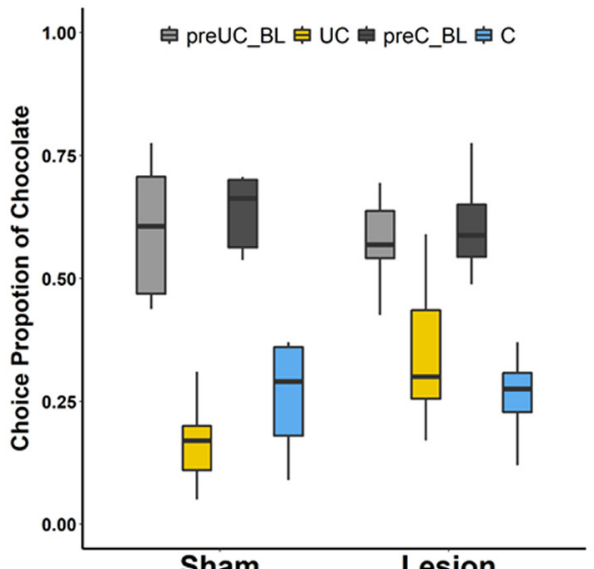

B

\section{Chocolate Elasticity}

๑ncompensated $\oslash$ Compensated

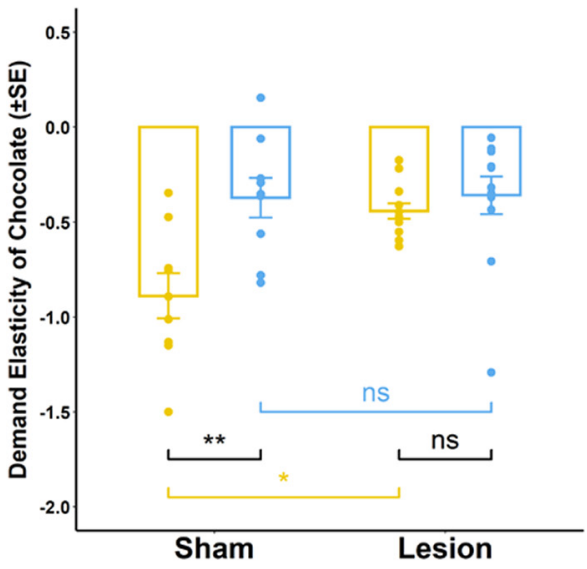

D

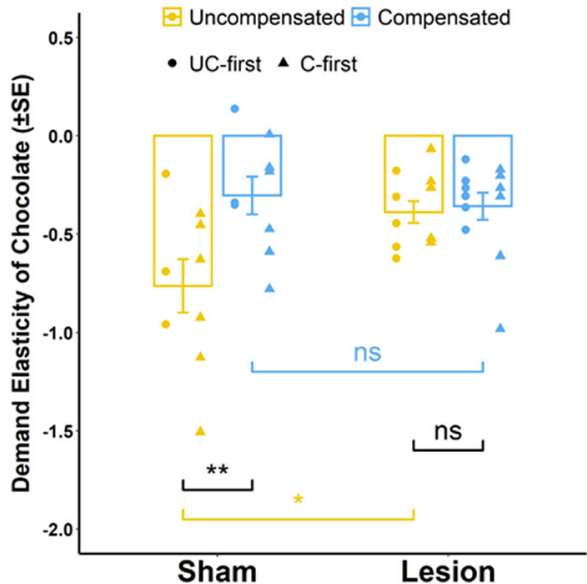

Figure 3. dACC lesions diminish budget effect on chocolate demand elasticity. $A$, The boxplots show the median choice proportions of chocolate milk in the baselines, uncompensated budget, and compensated budget conditions, averaged across the last two sessions per condition for the sham and lesioned rats. $\boldsymbol{B}$, Demand elasticity ( \pm SEM) of chocolate milk of the sham and lesion group. More negative demand elasticity indicates a stronger change in demand in response to price and/or budget changes. C, Proportion of chocolate choices in the paired baseline and budget conditions for the sham and lesion rats, averaged for the last two sessions. $D$, Demand elasticity of chocolate milk calculated from the paired baseline and budget conditions, averaged for the last two sessions. UC, uncompensated budget condition; C, compensated budget condition; BL, baseline; preUC_BL, baseline condition before the uncompensated budget condition; preC_BL, baseline condition before the compensated budget condition; UC-first, uncompensated budget condition first, compensated budget condition second (sham: $n=3$, lesion: $n=6$ ); C-first, compensated budget condition first, uncompensated second (sham: $n=6$, lesion: $n=6$ ); ${ }^{*} p<0.05,{ }^{* *} p<0.01$; n.s., not significant.

uncompensated budget conditions did not reach significance in the lesioned animals $\left(\varepsilon_{\text {Choco_Lesion_Compensated }}=-0.359 \pm 0.1\right.$, $\varepsilon_{\text {Choco__Lesion_Uncompensated }}=-0.443 \pm 0.04, t_{(11)}=-0.874$, $\left.p=0.356, \mathrm{BF}_{10}=0.386\right)$. We furthermore found that demand elasticity values were significantly different between sham and lesioned animals in the uncompensated condition $\left(t_{(19)}=\right.$ $\left.-3.418, p=0.007, \mathrm{BF}_{10}=35.232\right)$, but not in the compensated condition $\left(t_{(19)}=-0.095, p=0.355, \mathrm{BF}_{10}=0.395\right)$. This result is further support for our hypothesis (3) and corroborates the conclusion that dACC lesions selectively diminished the budget effect on demand elasticity for the preferred reward.

Demand elasticity quantified the change in consumption relative to the individual median baseline consumption across all three baseline sessions. As a robustness check, we ran additional analyses using local baselines. That is, for each rat, change in consumption was quantified relative to the consumption in the baseline immediately preceding the compensated or uncompensated budget condition. We found similar results on choice proportion (Fig. 3C) and demand elasticity (Fig. 3D). Paired and independent $t$ test revealed no significant differences between sham and lesioned animal in their baseline chocolate preferences (sham vs lesion for chocolate choices in the baseline preceding the uncompensated budget condition, preUC_BL, and baseline preceding the compensated budget condition, preC_BL, both $p>0.1$ ), as well as the baseline comparisons within each animal group (preUC_BL vs preC_BL for sham and lesion group, both $p>0.25)$. A $2 \times 2$ rmANOVA on chocolate elasticity including the order of budget conditions as a covariate confirmed a significant interaction effect between budget condition and lesion group $\left(F_{(1,16)}=8.128, p=0.011\right)$, and no significant order effect (order: $F_{(1,16)}=0.973, p=0.338$; order $\times$ budget: $F_{(1,16)}=0.355$, $p=0.559)$. A Bayesian rmANOVA also confirmed that a model $\left(\mathrm{BF}_{10}=22.014\right)$ containing budget condition, lesion and budget condition $\times$ lesion outperforms models with less factors and the model including order as an extra factor (all $\left.\mathrm{BF}_{10}<12.21\right)$ with a $\mathrm{BF}_{\text {incl }}=6.611$ for budget $\times$ lesion and $\mathrm{BF}_{\text {incl }}=0.549$ for the order effect.

Taken together, our pattern of results confirms our first three hypotheses. They suggest that all rats adjusted their demand of chocolate (and, by extension, vanilla; see below) to the respective price changes (i.e., effort discounting), and that demand elasticity of chocolate in response to price changes was different, in sham 
animals only, in the compensated compared with the uncompensated budget condition (i.e., budget effects on choice). Our data, furthermore, show that dACC lesions selectively diminished the budget effects on demand elasticity. Thus, it seems as if the sham rats considered the "purchasing power" of their budget above and beyond the net effort costs to obtain rewards, and that the lesioned rats selectively ignored the budget's purchasing power, although they still considered the rewards' net effort costs.

\section{dACC lesions abolish income but not substitution effects}

The previous analysis revealed that rats changed their demand of chocolate or vanilla when their price increased, or decreased respectively. Demand theory states that price effects on demand are because of both an income and a substitution effect. The income effect describes the change in commodity consumption that is caused by a change in the purchasing power of the budget (e.g., when prices increase or decrease, but the budget remains constant, as in the uncompensated budget condition). The substitution effect captures the change in consumption caused by consumers switching away from relatively more expensive to relatively cheaper alternatives. Both effects can be depicted by the shifts of choice bundle along the budget line under different experimental conditions (Fig. 4). A budget line represents the limit on all possible combinations of milk consumptions within the given budget and prices. The slope of budget line equals the price ratio of two reward commodities. Any change in the prices would result in altering the slope of budget line [e.g., from baseline to (un) compensated conditions]. An increase in the budget causes the budget line to shift outward, parallel to the original line if holding prices constant, indicating that a larger choice bundle can now be purchased (e. g., from uncompensated to compensated condition). The substitution effect can be analyzed by (1) compensating the budget to fit the previously established choice bundles according to the new prices and (2) observing whether consumers still deviate from re-selecting the original commodity bundle, i.e., by substituting the now more expensive commodity for the now cheaper one although they could theoretically repurchase their original bundle. In our case, most animal consumers preferred chocolate over vanilla rewards at baselines. Thus, here, pure substitution effects can be captured when the price of chocolate goes up and the budget is expanded (compensated condition), indicated by brown arrows in Figure $4 \mathrm{~A}$ showing a movement from point $\mathrm{BL}_{\text {sham }}$ to $\mathrm{C}_{\text {sham }}$ for sham group, and a movement from point $\mathrm{BL}_{\text {Lesion }}$ to $\mathrm{C}_{\text {Lesion }}$ for lesion group. If the budget is kept the same (uncompensated condition), an additional income effect, on top of the substitution effect, is thought to suppress chocolate choices even further. The income effect describes the change in commodity consumption that is caused by a change in the purchasing power of the budget (e.g., when prices increase or decrease, but the budget remains constant), indicated by dark green arrows in Figure $4 B$ showing a movement from point $C_{\text {sham }}$ to $\mathrm{UC}_{\text {sham }}$ for the sham group, and a movement from point $\mathrm{C}_{\text {Lesion }}$ to $\mathrm{UC}_{\mathrm{Lesion}}$ for the lesion group. We indeed found a non-zero income effect in our sham group (higher demand elasticity in the uncompensated than the compensated budget condition; see above and Fig. 5), suggesting that demand elasticity in these animals was driven by a combination of income and substitution effects. Since the dACC-lesioned animals, however, did not make significantly different reward bundle selections in the compensated and uncompensated budget conditions (no difference in demand elasticity between budget conditions; see above and Fig. 5), we conclude that dACC rats did show substitution effects, like the sham animals, but showed significantly reduced income effects. 
A

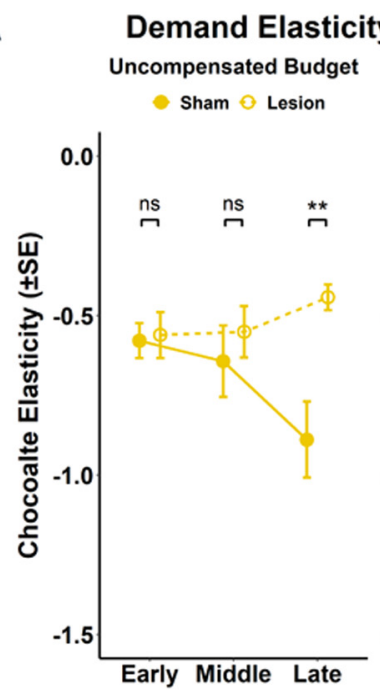

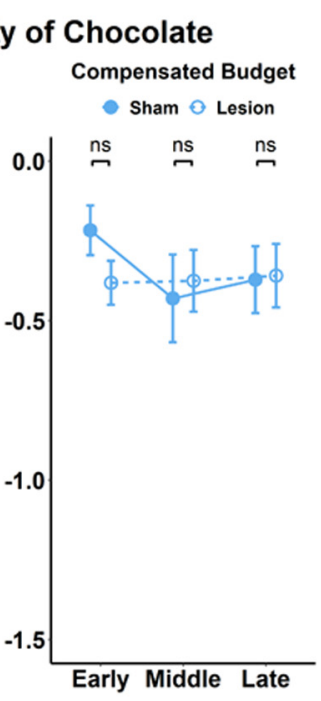

B

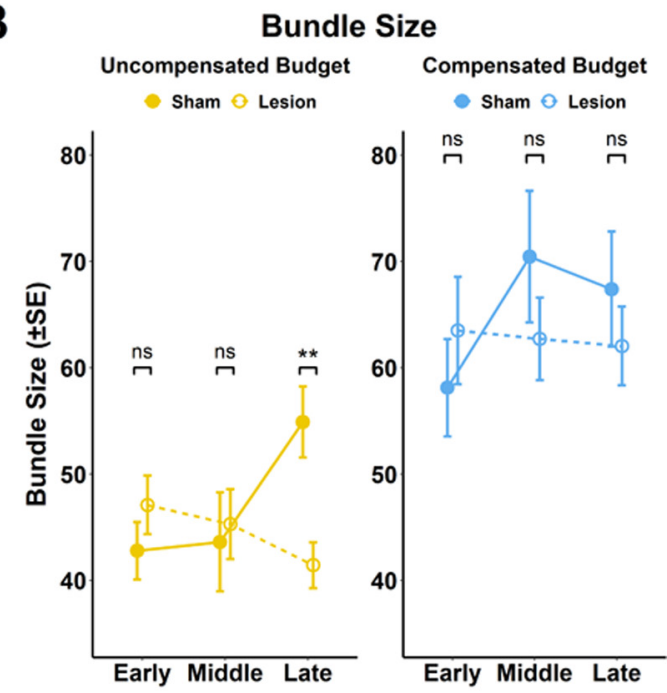

C
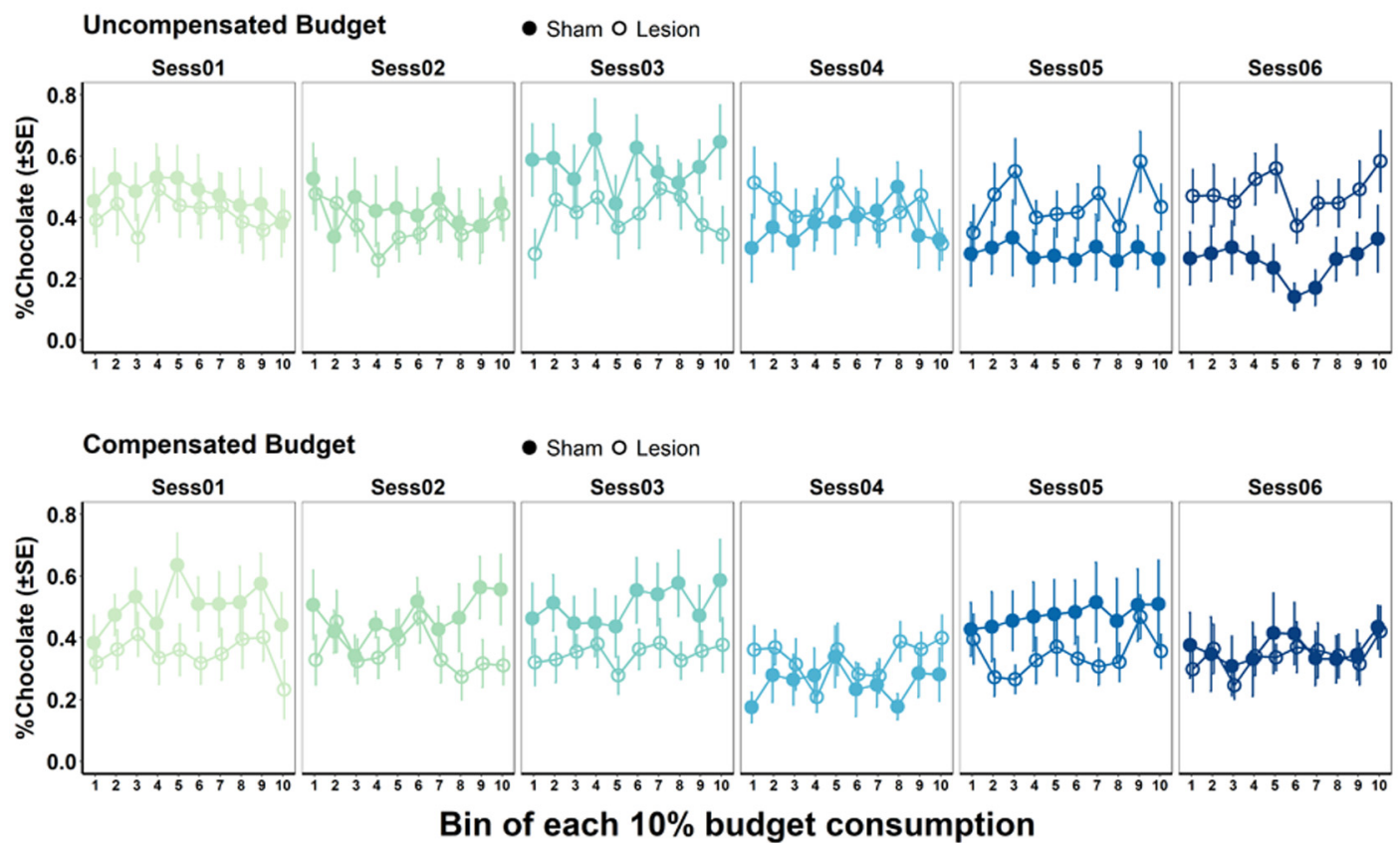

Figure 5. dACC lesions diminish session-based adaptation guiding budget effect. $A$, Mean demand elasticity ( \pm SEM) of chocolate in the early (sessions 1-2), middle (sessions 3-4), and late sessions (sessions 5-6) of the sham and lesion group. B. Mean total reward amounts (bundle size) earned in each session ( \pm SEM) in the early, middle and late sessions of the sham and lesion group. C, Choice proportion of chocolate ( \pm SEM) for each $10 \%$ bin of budget consumption across the six sessions in the uncompensated and compensated budget conditions; $* * p<0.01 ;$ n.S., not significant.

\section{What do the rats learn in this task?}

Our previous analysis has shown that, at the group level, both sham and lesion animals reduced their chocolate consumption when its price increased but purchasing power remained constant (i.e., under the substitution effect in the compensated budget condition), but that sham rats additionally considered their available budget during their cost/benefit value computation when their purchasing power was reduced (i.e., the additional income effect in the uncompensated budget condition). dACC lesions selectively abolished the income, but not the substitution effect on demand. Many questions remain: in contrast to price changes, which are experienced immediately, the budget extension is experienced necessarily only at the end of a session. Thus, how can the rats possibly know about the budget extensions or budget caps? In other words, how can rats possibly track, compute, process and update the purchasing value of their budget, and what is the precise role of dACC in these computations?

We reasoned that explicit knowledge about the budget size may not be necessary to explain the present pattern of results, as outlined in the following. In the baseline condition, given equal reward prices and a fixed budget, animals can always obtain a total of 40 units of milk per session. Rats, both in the sham and lesion group, prefer chocolate over vanilla when prices and, thus, efforts are equal. If a rat maintains its consumption strategy in the uncompensated condition when chocolate prices increase, and vanilla prices drop, they would earn fewer total rewards per session, for example, assume a rat spends its entire baseline budget on chocolate. It would, hence, obtain 40 units of chocolate, worth 80 NPs. In the uncompensated condition, the price of 
Table 1. Hypothetical examples illustrating that bundle size in the uncompensated and compensated budget condition depends on the baseline preferences for chocolate

\begin{tabular}{|c|c|c|c|c|c|c|c|c|c|}
\hline Example ID & $\begin{array}{l}\text { Baseline condition } \\
\text { Chocolate }\end{array}$ & $\begin{array}{l}\text { Uncompensated budget condition } \\
\text { Vanilla }\end{array}$ & $\begin{array}{l}\text { Compensated budget condition } \\
\text { Bundle size }\end{array}$ & Chocolate & Vanilla & Bundle size & Chocolate & Vanilla & Bundle size \\
\hline Rat A, high chocolate preference & 40 & 0 & 40 & 20 & 0 & 20 & 40 & 0 & 40 \\
\hline Rat $B$, medium chocolate preference & 30 & 10 & 40 & 15 & 20 & 35 & 30 & 10 & 40 \\
\hline Rat $C$, no preference for chocolate & 20 & 20 & 40 & 12 & 32 & 44 & 20 & 20 & 40 \\
\hline
\end{tabular}

The table shows the expected bundle composition and bundle size in the baseline, uncompensated and compensated budget conditions if rats maintained their baseline choice strategy after price/budget changes.

chocolate increases to 4 NPs and the budget remains at 80 NPs. If a rat perseverated on its baseline strategy of only purchasing chocolate, it would obtain a mere total of 20 reward units in this session, thus only half the amount of rewards than during baseline. Thus, there is an incentive to reduce chocolate consumption and increase vanilla consumption to avoid reducing the total amount of rewards earned in a session (the bundle size). This is true for the uncompensated budget condition, but not for the compensated budget condition where there is no pressure to adjust the consumption strategy (i.e., perseverating on a choice strategy in the compensated budget condition would yield the exact same chocolate and vanilla composition earned under baseline; compare Table 1).

Note that this is an extreme example, as rats rarely selected chocolate only during baseline sessions, but it was almost always the most frequently chosen reward. The predicted reduction in bundle size is directly related to the number of chocolate units consumed at baseline. To illustrate this, consider the following next hypothetical example (compare Table 1): at baseline, rat A consumes 40 units of chocolate (worth 80 NPs) and 0 units of vanilla (high chocolate preferences), rat B consumes 30 units of chocolate (worth $60 \mathrm{NPs}$ ) and 10 units of vanilla (worth 20 NPs; medium chocolate preference), and rat $\mathrm{C}$ consumes 20 units of chocolate (worth $40 \mathrm{NPs}$ ) and 20 units of vanilla (worth 40 NPs; indifferent between chocolate and vanilla). If a rat maintained its consumption strategy after the price change in the uncompensated budget condition, rat A would obtain 20 units of chocolate (worth $80 \mathrm{NPs}$ ) and 0 units of vanilla (bundle size 20 rewards; as explained above), rat $\mathrm{B}$ would obtain 15 units of chocolate (worth $60 \mathrm{NPs}$ ) and 20 units of vanilla (worth $20 \mathrm{NPs}$; bundle size 35 rewards), and rat $\mathrm{C}$ would obtain 10 units of chocolate (worth $40 \mathrm{NPs}$ ) and 40 units of vanilla (worth $40 \mathrm{NPs}$; bundle size 50 rewards). Hence, by perseverating on their baseline consumption strategy, rat $\mathrm{A}$ with a high baseline preference for chocolate would drastically reduce its bundle size in the uncompensated budget condition, rat B with medium baseline preference for chocolate would roughly maintain its bundle size after the price change, and rat $\mathrm{C}$ with a low preference for chocolate would even increase its bundle size.

However, by changing its strategy across uncompensated budget sessions after a price change, i.e., by gradually decreasing the proportion of chocolate choices and increasing the proportion of vanilla choices across sessions, a rat with medium-tostrong baseline preferences for chocolate could restore, or exceed, the total number of reward units earned relative to baseline. In other words, reducing chocolate and increasing vanilla consumption would maximize the bundle size or, by reverse logic, not replacing chocolate with vanilla would result in smaller-than-possible bundle sizes, and, thus, reduced session utility derived from reward bundles. Because the reduction in bundle size would be higher the larger the preference for chocolate in baseline sessions (if the baseline consumption strategy was maintained), the pressure to substitute expensive chocolate with cheaper vanilla in the uncompensated budget condition should be tightly linked to baseline chocolate preferences above and beyond mere price (or effort) discounting effects. In other words, the degree by which animals shift from chocolate to vanilla, i.e., cross-price elasticity estimates for vanilla (see Materials and Methods), should be correlated with the rats' individual baseline preferences for chocolate. Again, this is true for the uncompensated budget condition, but not for the compensated budget condition because of the above-mentioned lack of pressure to adjust the consumption strategy (compare Table 1).

We expect that, after a price change in the uncompensated budget condition, rats will, at first, continue their baseline strategy, which will yield a reduced reward bundle size relative to baseline. Sham rats will adjust their strategy by reducing their chocolate consumption and increasing their vanilla consumption, dependent also on their individual baseline preferences for chocolate, resulting in a gradual increase of bundle size across sessions after a price and budget reversal. The pressure for this strategy change will be higher in the uncompensated than the compensated budget condition. dACC-lesioned animals also respond to the price changes, but will fail to change their strategy across sessions in either budget condition.

Thus, in short, this theory assumes that sham rats maximize bundle utility (the utility of the total rewards obtained by the end of a session) which is a function of the bundle composition (e.g., more chocolate is better than more vanilla) and bundle size (more reward of any kind is better than less reward). Sham rats flexibly adjust their choice strategy across sessions after a price/ budget change to maximize the bundle utility, especially bundle size, but dACC-lesioned animals fail to do so.

This theory makes several predictions. First, in the uncompensated, but to a lesser extent in the compensated budget condition, sham rats, but not lesioned rats, learn to reduce their chocolate consumption across sessions after a price change, which should go along with a gradual increase in total rewards earned; i.e., demand elasticity of chocolate as well as bundle size should evolve across sessions after a price change in the sham rats, but not the dACC-lesioned rats, reflecting the gradual adjustment of the choice strategy. Second, rats should gradually shift from expensive chocolate to cheaper vanilla across sessions after a price change, but the extent of this shift should depend on the individual baseline preferences for chocolate; i.e., cross-price elasticity estimates for vanilla (as a proxy for the extent of the shift from chocolate to vanilla) should be correlated with baseline preferences for chocolate in the uncompensated budget condition in the sham, but not the dACC-lesioned animals.

To address the first prediction, we calculated the demand elasticity of chocolate in blocks of two sessions (early: sessions 1-2; middle: sessions 3-4; late: sessions 5-6) in each budget condition and ran a mixed ANOVA on session block (early vs middle vs late, within-subjects) and lesion (sham vs lesion; between-subjects) for the uncompensated and compensated budget condition. In the uncompensated condition, we found a 
main effect of lesion $\left(F_{(1,19)}=6.16, p=0.023\right.$, partial $\left.\eta^{2}=0.12\right)$ and a significant interaction between session block and lesion $\left(F_{(2,38)}=4.545, p=0.017\right.$, partial $\left.\eta^{2}=0.121\right)$. A Bayesian rmANOVA yielded a $\mathrm{BF}_{10}=1.577$ for the full model as well as a $\mathrm{BF}_{\text {incl }}=5.137$ for block $\times$ lesion, thus supporting evidence for an interaction effect. Breaking down this interaction, we found that, compared with lesioned rats, sham rats exhibited a significantly more elastic demand for chocolate in the late session block $\left(t_{(17)}=3.873, p=0.004\right)$, but not in the early sessions or middle block (both $p>0.497$ ), suggesting that the difference in demand elasticity between sham and lesioned rats evolved across sessions (Fig. 5A). In the compensated budget condition, we found neither a significant main effect of lesion $\left(F_{(1,19)}=0.103, p=0.752\right.$, partial $\left.\eta^{2}=0.003\right)$, nor an interaction effect with session block $\left(F_{(2,38)}=0.976, p=0.386\right.$, partial $\left.\eta^{2}=0.024\right)$. The Bayesian rmANOVA confirmed the absence of this effect by reporting a $\mathrm{BF}_{10}=0.03$ for the full model as well as a $\mathrm{BF}_{\text {incl }}=0.374$ for session block $\times$ lesion interaction. Thus, this analysis suggests that, in accordance with the first prediction of our theory, demand elasticity of chocolate became more elastic across sessions in the sham rats in the uncompensated, but not in the compensated budget condition. There was no evidence for a shift in demand elasticity in any budget condition in the dACClesioned rats.

We next asked whether the total number of chocolate and vanilla rewards earned per session, i.e., the overall bundle size, changed across sessions in the two budget conditions (Fig. 5B). We computed the average bundle size in blocks of two sessions and ran a similar two-way mixed ANOVA on session block (early, middle, and late) and lesion (lesion vs sham) on bundle size for the uncompensated and compensated budget condition. We found a significant interaction effect between session block and lesion $\left(F_{(2,38)}=5.065, p=0.011\right.$, partial $\left.\eta^{2}=0.138\right)$ in the uncompensated budget condition. Breaking down this interaction effect, sham rats obtained a much larger total reward amount in the late sessions than lesioned animals $(p=0.002)$, but this difference was absent in the early or middle sessions (both $p>0.29$ ). This suggests that, in the uncompensated condition, bundle size gradually increased across sessions in the sham animals, but not the lesioned animals. The Bayesian rmANOVA reported $\mathrm{BF}_{10}=0.594$ for the full model suggesting insufficient evidence in favor of the model; however, we obtained a $\mathrm{BF}_{\text {incl }}=$ 8.194 for session block $\times$ lesion in support of the interaction effect. In the compensated budget condition, we found neither main effects nor interaction effects between block and lesion (all $p>0.327)$ on total bundle size. Bayesian rmANOVA yielded a $\mathrm{BF}_{10}=0.036$ for the full model as well as a $\mathrm{BF}_{\text {incl }}=0.087$ for session block $\times$ lesion.

The analysis so far suggests that demand elasticity and bundle size changed with learning across sessions. To explore whether rats also showed within-session learning, we quantified the change in chocolate choices within sessions. To this end, we calculated the choice proportion of chocolate in each $10 \%$ bin of the total budget for each budget session (Fig. 5C) and ran a 2 (sham vs lesion $) \times 10$ (10 bins) rmANOVA for each condition. In the last session (Sess06) of the uncompensated budget condition, we found a main effect of lesion on chocolate choices $\left(F_{(1,19)}=8.23\right.$, $p=0.01)$ but no effect of bin $\left(F_{(1,9)}=1.39, p=0.24\right)$ nor aninteraction effect of lesion $\times$ bin $\left(F_{(1,9)}=0.315, p=0.969\right)$. No significant effects were found in any other session of the uncompensated and compensated budget condition. To quantify the evidence in favor of the idea that lesion effects on chocolate choices developed within and across sessions, we ran a Bayesian rmANOVA for each session data. The Bayesian rmANOVA confirmed that the model containing lesion effect only $\left(\mathrm{BF}_{10}=\right.$ 5.243) outperformed other models for the uncompensated Sess06. We also found an increased $\mathrm{BF}_{10}$ of this model for the early uncompensated sessions (Sess01: $\mathrm{BF}_{10}=0.549$; Sess02: $\mathrm{BF}_{10}=0.508 ;$ Sess03: $\mathrm{BF}_{10}=0.785 ;$ Sess04: $\mathrm{BF}_{10}=0.78 ;$ Sess05: $\left.\mathrm{BF}_{10}=0.948\right)$. The $\mathrm{BF}_{10}$ can be interpreted as the likelihood of the observed data explained by the test model compared with the alternative model (i.e., best model), therefore, the increasing $\mathrm{BF}_{10}$ indicates an emerging lesion effect on chocolate choices across uncompensated sessions, but absence of any within-session adaptations. We therefore conclude that learning likely happened across sessions, but not within sessions.

Thus, in support of the first prediction, demand elasticity and bundle size increased across sessions in the uncompensated budget condition, when the pressure to adjust the choice strategy was high (compare Table 1), but less so in the compensated budget condition where the pressure for strategy adjustment was lower. Importantly, this pattern was found in the sham rats, but not in the lesioned rats.

Our second hypothesis predicted that the tendency to replace expensive chocolate with cheaper vanilla in the uncompensated budget condition should be correlated with the rats' individual baseline preferences for chocolate. To test this hypothesis, we ran a four-way multiple linear regression on cross-price elasticity of vanilla with the factors baseline chocolate preference ("scaled_choc_base", Table 2), lesion (lesion vs sham), session block (early, middle, late), and budget condition (compensated vs uncompensated). We found a main-effect linear relationship between baseline chocolate preferences and vanilla cross-price elasticity $\left(\beta=-0.056 \pm 0.021, t_{(17)}=2.70, p=0.015\right)$, a main effect of lesion $\left(\beta=0.329 \pm 0.108, t_{(17)}=3.04, p=0.007\right)$ and a main effect of condition $\left(0.431 \pm 0.083, t_{(85)}=5.22, p=0.000\right)$ on vanilla cross-price elasticity. Importantly, we also found a significant four-way interaction between lesion groups, budget condition, session block, and baseline chocolate preference $(-0.144 \pm 0.050$, $\left.t_{(85)}=-2.90, p=0.005\right)$. To facilitate the interpretation of this negative four-way interaction, we refer to our directed hypothesis that we expected to find a difference in the relationship between baseline chocolate preference and vanilla cross-price elasticity between sham and lesioned animals in the late block of sessions and in the uncompensated, but not the compensated budget condition. Indeed, when restricting the linear model to the vanilla cross-price elasticity estimates in the uncompensated budget condition in the late block, we found a significant positive relationship between baseline chocolate preference and vanilla cross-price elasticity ( $\beta$ $\left.=0.070 \pm 0.016, t_{(17)}=4.34, p=0.001\right)$ and a significant negative interaction between lesion group and the slope of this relationship $\left(\beta=-0.061 \pm 0.023, t_{(17)}=-2.597, p=0.017\right)$. This suggests that the correlation between baseline chocolate preference and vanilla cross-price elasticity was significantly positive in the sham animals, but almost absent in the lesioned animals (Fig. 6). Notably, this slope-by-lesion interaction was absent in the compensated budget condition and in the early and middle block in either budget condition (see Table 2).

To put this complex pattern of results in simple terms: all rats reduced their consumption of chocolate and they increased their consumption of vanilla in response to the price changes (price effect on demand), and all rats substituted the more expensive chocolate with cheaper vanilla after the price change (substitution effect). But the reduction in chocolate consumption was stronger in the sham than the lesioned rats (diminished income 
effects after dACC lesions), going along with less pronounced chocolate-to-vanilla substitution in the lesioned rats (chocolate-preference-dependent cross-price elasticity of vanilla). Sham, but not dACC rats, gradually learned the adequate, baseline-preferencedependent level of chocolate-to-vanilla-shifts across sessions after a price and/or budget change (the size and composition of the total number of rewards earned in a session).

These findings are in line with the predictions of our theory outlined above. It supports the intriguing possibility that the currency that is maximized by the rats is the utility of the reward bundle obtained in a session, which is a function of the composition of the bundle (more chocolate is better than more vanilla) as well its size (more reward of any type is better than less reward). This session-wise computation of bundle utility is hypothesized to be dACC dependent.

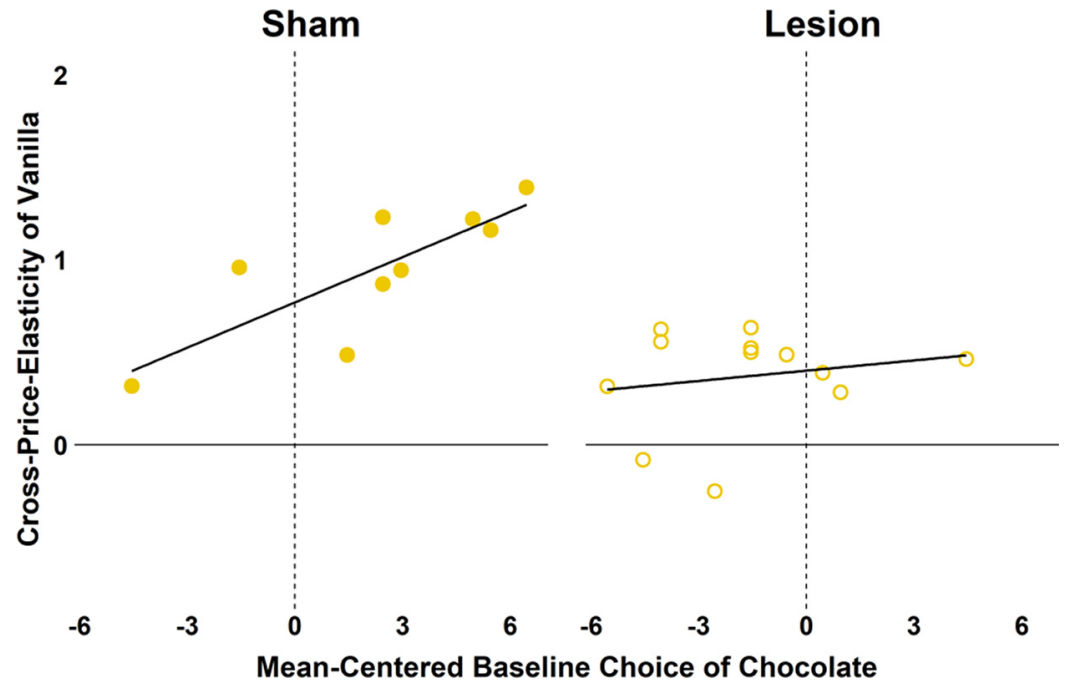

Figure 6. dACC lesions impaired reward substitution under the budget constraint in the late sessions. Cross-price elasticity of vanilla depended on the baseline choices for chocolate in the sham animals but not in $\mathrm{dACC}-$ lesioned animals. See text for further explanation.

\section{Discussion}

Economic theory can be applied to describe and predict the choices of animal consumers (Kagel et al., 1975, 1981; Chen et al., 2006; Freidin and Kacelnik, 2011; Shizgal, 2012; PastorBernier et al., 2017). Several studies revealed that rats are not only sensitive to the effort (the price) required to obtain a reward, but also to the available budget, i.e., the total number of responses available to "pay" for rewards. For example, we have shown (van Wingerden et al., 2015) that rats reduce their demand of a commodity when its price increased, but they responded to the price changes less strongly when the budget was adjusted to compensate for price inflation. That is, the rats behaved as if they considered the purchasing power of their budget in their cost-benefit evaluation.

Here, we demonstrate that bilateral lesions of dACC diminish this budget effect on demand for rewards. In our experiment, rat consumers made NPs to obtain chocolate or vanilla milk rewards. In three experimental conditions, we manipulated the effort requirement, i.e., the number of NPs necessary to obtain a reward, and the budget, i.e., the total amount of NPs available in a session to spend on rewards. In the baseline condition, the budget was fixed at $80 \mathrm{NPs}$, and chocolate and vanilla milk cost 2 NPs each. In the uncompensated budget condition, we increased the price of chocolate to $4 \mathrm{NPs}$, reduced the price of vanilla to one NP and kept the budget constant at 80 NPs. In the compensated budget condition, chocolate and vanilla prices were identical to the uncompensated budget condition, but we increased the budget according to the Slutsky equation (Slutsky and Ragusa, 2012) to compensate for the price change and allow animals to re-select the same chocolate-vanilla bundle chosen during the baseline condition.

We found that all rats adjusted heir demand for reward in response to price changes, i.e., they showed effort-discounting, but in sham-lesioned rats, demand elasticity was less pronounced in the compensated than the uncompensated budget condition, replicating the budget effect on demand elasticity previously shown (Kagel et al., 1975; van Wingerden et al., 2015). dACClesioned animals also preferred chocolate over vanilla in the baseline condition, similar to sham-lesioned animals, and they equally adjusted their demand for rewards to the price changes.
Table 2. Coefficients of mixed-effect model on cross-price elasticity of vanilla milk

\begin{tabular}{lcrrr}
\hline Predictor & Coefficient & SE & $t$ value & $p$ value \\
\hline Intercept) & 0.092 & 0.083 & 1.115 & 0.268 \\
scaled_choc_base & 0.056 & 0.021 & 2.699 & 0.015 \\
condition & 0.431 & 0.082 & 5.221 & 0.000 \\
middle & 0.327 & 0.156 & 2.098 & 0.039 \\
late & 0.213 & 0.152 & 1.396 & 0.166 \\
lesion & 0.329 & 0.108 & 3.044 & 0.007 \\
scaled_choc_base $\times$ condition & -0.031 & 0.021 & -1.486 & 0.141 \\
scaled_choc_base $\times$ middle & -0.051 & 0.039 & -1.301 & 0.197 \\
scaled_choc_base $\times$ late & -0.026 & 0.038 & -0.679 & 0.499 \\
condition $\times$ middle & -0.216 & 0.153 & -1.412 & 0.162 \\
condition $\times$ late & 0.050 & 0.135 & 0.369 & 0.713 \\
scaled_choc_base $\times$ lesion & -0.033 & 0.030 & -1.081 & 0.295 \\
condition $\times$ lesion & -0.236 & 0.108 & -2.185 & 0.032 \\
middle $\times$ lesion & -0.239 & 0.204 & -1.171 & 0.245 \\
late $\times$ lesion & -0.112 & 0.199 & -0.563 & 0.575 \\
scaled_choc_base $\times$ condition $\times$ middle & 0.030 & 0.038 & 0.784 & 0.435 \\
scaled_choc_base $\times$ condition $\times$ late & 0.048 & 0.034 & 1.403 & 0.164 \\
scaled_choc_base $\times$ condition $\times$ lesion & 0.040 & 0.030 & 1.327 & 0.188 \\
scaled_choc_base $\times$ middle $\times$ lesion & 0.107 & 0.057 & 1.872 & 0.065 \\
scaled_choc_base $\times$ late $\times$ lesion & 0.100 & 0.056 & 1.780 & 0.079 \\
condition $\times$ middle $\times$ lesion & 0.044 & 0.200 & 0.219 & 0.827 \\
condition $\times$ late $\times$ lesion & -0.306 & 0.176 & -1.736 & 0.086 \\
scaled_choc_base $\times$ condition $\times$ middle $\times$ lesion & -0.130 & 0.056 & -2.321 & 0.023 \\
scaled_choc_base $\times$ condition $\times$ late $\times$ lesion & -0.144 & 0.050 & -2.902 & 0.005 \\
\hline
\end{tabular}

Yet, there was no difference in their demand elasticity between the two budget conditions. Thus, we conclude that dACC lesions selectively diminished the budget effects on demand elasticity while leaving the capacities for basic reward valuation, price/ effort sensitivity, behavioral flexibility and general motor behavior intact. Thus, while our sham rats behaved as if they considered the purchasing power of their budget above and beyond the rewards' nominal costs, as shown before (van Wingerden et al., 2015), dACC-lesioned rats behaved as if they selectively ignored the purchasing power of their budget. Hence, ACC seems to be necessary to integrate the budget information into the cost-benefit calculation. 
Previous studies that identified the budget effect on demand elasticity (Kagel et al., 1975; van Wingerden et al., 2015) were silent about the possible underlying cognitive mechanisms. That is, it is unknown how rats could possibly represent and consider the budget during their cost-benefit calculations. It is unlikely that rats have a numerical representation of their budget, and it is equally unlikely that they track an alternative budget metric, such as the time available to purchase rewards. So, the question remains what information rats mentally process when they consider budget and purchasing power. We reasoned that the behavioral deficits after dACC lesions might shed light on this question.

We found that, while bundle size and demand elasticity for chocolate increased across sessions in the uncompensated, but not in the compensated budget condition in the sham animals, no session-wise change in bundle size or bundle composition was found in the dACC-lesioned animals. This result raises the intriguing possibility that the acquired change in the budget-dependent consumption strategy across sessions lies at the core of the budget effect on demand elasticity, as outlined in the following.

At baseline, rats reveal a preference for a particular bundle composition, typically consisting of a larger proportion of chocolate and a smaller proportion of vanilla. If the rats maintained their choice strategy in the uncompensated budget condition when chocolate prices increase, vanilla prices drop and the budget remains fixed, the bundle size would shrink as the rats would purchase less chocolate. The shrinkage in bundle size should be larger the higher the baseline preference for chocolate. However, by changing the strategy in the uncompensated budget condition, i.e., by substituting expensive chocolate with cheaper vanilla, the rats would restore, or exceed, the total number of reward units earned in a baseline session (compare Table 1). Thus, the desire to maximize bundle size might underlie strategy selection in the uncompensated budget condition. Importantly, because of the budget adjustment to the price changes, there is less pressure for strategy adaptation in the compensated budget condition. In support of this theory, we found that sham rats gradually learned to reduce their chocolate consumption and increase their vanilla consumption across sessions in the uncompensated, but less so in the compensated budget condition, resulting in a session-wise increase in bundle size. The magnitude of the chocolate-to-vanilla shift was dependent on the strength of baseline preference for chocolate. This pattern was not found in dACC-lesioned animals.

Put together, we argue that there is no need to assume a numerical budget representation to explain the budget effects on demand elasticity. Instead, our results imply that rats compute and maximize a session-wise satisfaction signal that represents the utility of the reward bundle obtained in a session. This bundle utility is assumed to be a function of the total amount of rewards earned in a session (more reward is better) and the composition of the reward bundle (more chocolate is better than more vanilla). The session-wise computation of a satisfaction signal requires local non-satiation (rats consistently want more reward) and strong monotonicity of reward value (more of a good thing is better than less of a good thing), assumptions that are realistic and plausible. Because dACC-lesioned rats failed to increase bundle size in the uncompensated budget condition, and, hence, failed to maximize the session-wise bundle utility, we conclude that dACC integrity is necessary to track and update the reward satisfaction derived from the choice bundle consumed in a session.
Our theory makes the interesting prediction that ACC drives higher-order learning by encoding a session-wise prediction error that signals the discrepancy between expected and obtained bundle utility. Prediction errors should be larger after price and/ or budget changes. There is ample evidence that ACC indeed encodes positive and negative prediction errors in more simple tasks (Amiez et al., 2005; Behrens et al., 2007; Seo and Lee, 2007; Bryden et al., 2011; Hayden et al., 2011b; Hyman et al., 2017; Arulpragasam et al., 2018), but it is elusive if this also holds for the complex, session-wise bundle utility value proposed here. In a more general sense, our data are consistent with, and expand on, the idea that ACC is important for computing the relative value of changing the current decision policy for an alternative, potentially better policy, reminiscent of its role in tracking the value of alternative courses of action during optimal foraging (Hayden et al., 2011a; Kolling et al., 2014; Costa and Averbeck, 2015). Future electrophysiological studies might shed more light on this possibility.

Our results suggest that dACC deficits might not simply cause an altered effort tolerance, but rather a failure to integrate multiple information sources across sessions to compute a representation of session utility, or task value, respectively (Kennerley et al., 2006; Walton et al., 2007; Hillman and Bilkey, 2010; Heilbronner and Hayden, 2016; Kolling et al., 2016). A similar contextual concept of "task value" was introduced in a previous study (Amiez et al., 2006), where ACC neural activity did not track reward values on a trial-by-trial basis, but the maximum averaged reward across trials. Similar to our findings, the authors also reported that ACC inactivation induced a non-optimal strategy in animals' foraging behavior. Overall, our data are in line with a model according to which ACC plays a role in the hierarchical organization of effortful behavior (Holroyd and McClure, 2015). According to this model, ACC selects and integrates high-level reward, cost and task information across trials. By using this information, ACC is in a position to perform behavioral policy evaluations and updating and can regulate the degree of control over downstream areas based on whether rewards are considered better or worse than a benchmark reward value criterion, such as, here, the session-wise bundle utility.

In conclusion, we found that sham rats behaved as if they considered the purchasing power of their budget above and beyond the rewards' nominal costs, dACC-lesioned rats behaved as if they selectively ignored the purchasing power of their budget. Our results offer initial mechanistic insight on how budget affects the selection of choice strategies, and how dACC lesions interfere with this process. As animal models are an invaluable instrument to investigate the neurobiological mechanisms of psychiatric disorders (Ward et al., 2011; Izquierdo et al., 2019), our paradigm is a valuable addition to the existing toolboxes to probe multi-dimensional cost-benefit computations in adaptive behavior.

\section{References}

Amiez C, Joseph JP, Procyk E (2005) Anterior cingulate error-related activity is modulated by predicted reward. Eur J Neurosci 21:3447-3452.

Amiez C, Joseph JP, Procyk E (2006) Reward encoding in the monkey anterior cingulate cortex. Cereb Cortex 16:1040-1055.

Arulpragasam AR, Cooper JA, Nuutinen MR, Treadway MT (2018) Corticoinsular circuits encode subjective value expectation and violation for effortful goal-directed behavior. Proc Natl Acad Sci USA 115:E5233E5242.

Behrens TE, Woolrich MW, Walton ME, Rushworth MF (2007) Learning the value of information in an uncertain world. Nat Neurosci 10:12141221. 
Bryden DW, Johnson EE, Tobia SC, Kashtelyan V, Roesch MR (2011) Attention for learning signals in anterior cingulate cortex. J Neurosci 31:18266-18274.

Chen MK, Lakshminarayanan V, Santos LR (2006) How basic are behavioral biases? Evidence from capuchin monkey trading behavior. J Polit Econ 114:517-537.

Costa VD, Averbeck BB (2015) Frontal-parietal and limbic-striatal activity underlies information sampling in the best choice problem. Cereb Cortex 25:972-982.

Croxson PL, Walton ME, O’Reilly JX, Behrens TE, Rushworth MF (2009) Effort-based cost-benefit valuation and the human brain. J Neurosci 29:4531-4541.

Deaton A, Muellbauer J (1980) Economics and consumer behavior. Cambridge: Cambridge University Press.

Freidin E, Kacelnik A (2011) Rational choice, context dependence, and the value of information in European starlings (Sturnus vulgaris). Science 334:1000-1002.

Hayden BY, Pearson JM, Platt ML (2011a) Neuronal basis of sequential foraging decisions in a patchy environment. Nat Neurosci 14:933-939.

Hayden BY, Heilbronner SR, Pearson JM, Platt ML (2011b) Surprise signals in anterior cingulate cortex: neuronal encoding of unsigned reward prediction errors driving adjustment in behavior. J Neurosci 31:4178-4187.

Heilbronner SR, Hayden BY (2016) Dorsal anterior cingulate cortex: a bottom-up view. Annu Rev Neurosci 39:149-170.

Hillman KL, Bilkey DK (2010) Neurons in the rat anterior cingulate cortex dynamically encode cost-benefit in a spatial decision-making task. J Neurosci 30:7705-7713

Hillman KL, Bilkey DK (2012) Neural encoding of competitive effort in the anterior cingulate cortex. Nat Neurosci 15:1290-1297.

Holroyd CB, McClure SM (2015) Hierarchical control over effortful behavior by rodent medial frontal cortex: a computational model. Psychol Rev 122:54-83.

Hyman JM, Holroyd CB, Seamans JK (2017) A novel neural prediction error found in anterior cingulate cortex ensembles. Neuron 95:447-456.e3.

Izquierdo A, Aguirre C, Hart EE, Stolyarova A (2019) Rodent models of adaptive value learning and decision-making. Methods Mol Biol 2011:105-119.

JASP Team J (2019) JASP (version 0.11.1) [Computer software]

Kagel JH, Rachlin H, Green L, Battalio RC, Basmann RL, Klemm WR (1975) Experimental studies of consumer demand behavior using laboratory animals. Econ Inq 13:22-38.

Kagel JH, Battalio RC, Rachlin H, Green L (1981) Demand curves for animal consumers. Q J Econ 96:1-15.

Kennerley SW, Wallis JD (2009) Evaluating choices by single neurons in the frontal lobe: outcome value encoded across multiple decision variables. Eur J Neurosci 29:2061-2073.

Kennerley SW, Walton ME, Behrens TE, Buckley MJ, Rushworth MF (2006) Optimal decision making and the anterior cingulate cortex. Nat Neurosci 9:940-947.

Keysers C, Gazzola V, Wagenmakers EJ (2020) Using Bayes factor hypothesis testing in neuroscience to establish evidence of absence. Nat Neurosci 23:788-799.

Klein-Flügge MC, Kennerley SW, Friston K, Bestmann S (2016) Neural signatures of value comparison in human cingulate cortex during decisions requiring an effort-reward trade-off. J Neurosci 36:10002-10015.

Kolling N, Behrens TE, Mars RB, Rushworth MF (2012) Neural mechanisms of foraging. Science 336:95-98.
Kolling N, Wittmann M, Rushworth MF (2014) Multiple neural mechanisms of decision making and their competition under changing risk pressure. Neuron 81:1190-1202.

Kolling N, Wittmann MK, Behrens TE, Boorman ED, Mars RB, Rushworth MF (2016) Value, search, persistence and model updating in anterior cingulate cortex. Nat Neurosci 19:1280-1285.

Kurzban R, Duckworth A, Kable JW, Myers J (2013) An opportunity cost model of subjective effort and task performance. Behav Brain Sci 36:661679.

Laughlin SB, de Ruyter van Steveninck RR, Anderson JC (1998) The metabolic cost of neural information. Nat Neurosci 1:36-41.

Lee MD, Wagenmakers EJ (2014) Bayesian cognitive modeling: a practical course. Cambridge: Cambridge University Press.

Maechler M, Stahel W, Ruckstuhl A, Keller C, Halvorsen K, Hauser A, Buser C (2020) Package 'sfsmisc'. Available at https://github.com/mmaechler/ sfsmisc.

Morey RD, Romeijn JW, Rouder J (2016) The philosophy of Bayes factors and the antification of statistical evidence. J Math Psychol 72:6-18.

Pastor-Bernier A, Plott CR, Schultz W (2017) Monkeys choose as if maximizing utility compatible with basic principles of revealed preference theory. Proc Natl Acad Sci USA 114:E1766-E1775.

Paxinos G, Watson C (2006) The rat brain in stereotaxic coordinates: hard cover edition. Elsevier.

Pinheiro J, Bates D, DebRoy S, Sarkar D, Team RC (2007) Linear and nonlinear mixed effects models. R package version 3:1-89.

Porter BS, Hillman KL, Bilkey DK (2019) Anterior cingulate cortex encoding of effortful behavior. J Neurophysiol 121:701-714.

Prévost C, Pessiglione M, Météreau E, Cléry-Melin ML, Dreher JC (2010) Separate valuation subsystems for delay and effort decision costs. J Neurosci 30:14080-14090.

Ripley B, Venables B, Bates D, Hornik K, Gebhardt A, Firth D, Ripley M (2013) Package 'mass'. Cran R 538

Rudebeck PH, Walton ME, Smyth AN, Bannerman DM, Rushworth MF (2006) Separate neural pathways process different decision costs. Nat Neurosci 9:1161-1168.

Seo H, Lee D (2007) Temporal filtering of reward signals in the dorsal anterior cingulate cortex during a mixed-strategy game. J Neurosci 27:83668377.

Shizgal P (2012) Scarce means with alternative uses: Robbins' definition of economics and its extension to the behavioral and neurobiological study of animal decision making. Front Neurosci 6:20.

Slutsky EE, Ragusa O (2012) On the theory of the budget of the consumer. G Econ Ann Econ 71:173-200.

van Wingerden M, Marx C, Kalenscher T (2015) Budget constraints affect male rats' choices between differently priced commodities. PLoS One 10: e0129581.

Vassena E, Krebs RM, Silvetti M, Fias W, Verguts T (2014) Dissociating contributions of ACC and vmPFC in reward prediction, outcome, and choice. Neuropsychologia 59:112-123.

Walton ME, Bannerman DM, Rushworth MF (2002) The role of rat medial frontal cortex in effort-based decision making. J Neurosci 22:1099611003 .

Walton ME, Croxson PL, Behrens TE, Kennerley SW, Rushworth MF (2007) Adaptive decision making and value in the anterior cingulate cortex. Neuroimage 36 [Suppl 2]:T142-T154.

Ward RD, Simpson EH, Kandel ER, Balsam PD (2011) Modeling motivational deficits in mouse models of schizophrenia: behavior analysis as a guide for neuroscience. Behav Processes 87:149-156. 